\title{
Editorial: Metabolically Healthy and Unhealthy Obese Children and Adolescents
}

\author{
Claudio Chiesa ${ }^{1 *}$, Lucia Pacifico ${ }^{2}$, Bo Xi ${ }^{3}$ and Cristina Cadenas-Sanchez ${ }^{4}$ \\ ${ }^{1}$ Institute of Translational Pharmacology, National Research Council (CNR), Rome, Italy, ${ }^{2}$ Department of Maternal and Child \\ Health, Sapienza University of Rome, Rome, Italy, ${ }^{3}$ Department of Epidemiology, School of Public Health, Shandong \\ University, Jinan, China, ${ }^{4}$ Institute for Innovation \& Sustainable Development in Food Chain (IS-FOOD), Public University of \\ Navarra, Pamplona, Spain
}

Keywords: metabolic phenotypes, obesity, children, organ damage, nonalcoholic fatty liver disease

Editorial on the Research Topic

Metabolically Healthy and Unhealthy Obese Children and Adolescents

\section{INTRODUCTION}

It is well established that pediatric obesity is closely associated with an increased risk of metabolic diseases, all of which lead to marked increase in cardiovascular disease (CVD) events in adulthood (1). Therefore, there is a need for an early identification and treatment of youths who are at greater risk of developing obesity-related complications (2). A growing interest has been raised regarding a subgroup of obese individuals, including children, who are metabolically healthy, for whom the term "metabolically healthy obese (MHO)" has been proposed $(3,4)$. Since MHO in adults has its origin in the childhood years (5), stratification of obese youths, based on metabolic heath status, might be of crucial importance to improve in early ages prevention strategies for cardiometabolic diseases.

This Research Topic draws together seven original articles, and one comprehensive review (Vukovic et al.) summarizing the current knowledge on the definition, epidemiology, predictors, underlying mechanisms, and treatment outcomes of the MHO phenotype in the pediatric population.

\section{CRITERIA TO IDENTIFY MHO AND EPIDEMIOLOGY}

In recent years, a voluminous literature has accumulated, advocating different definitions of $\mathrm{MHO}$ in children. However, the use of non-harmonized definitions of MHO has been a major problem, making it difficult to compare prevalence data among studies. As such, the MHO prevalence has been estimated to range between $7 \%$ and $21 \%$ in youths, notably between $3 \%$ and $87 \%$ in those with overweight/obesity (Vukovic et al.). In one international cohort including 3,497 children, 4.5\% were classified as MHO according to the modified National Cholesterol Education Program (NCEP) criteria, while 8.2\% according to the modified International Diabetes Federation criteria (6). Yet, in a national survey of 4,200 Iranian children, the prevalence of $\mathrm{MHO}$ was $10.4 \%$ according to the modified NCEP criteria (7). For these reasons, an international panel of 46 experts has recently convened to create consensus ( $\geq 80 \%$ agreement) on an evidence-based definition of MHO in children (8). The experts agreed on using the World Health Organization body mass index criteria to assess weight status, and including high- 
density lipoprotein cholesterol $>40 \mathrm{mg} / \mathrm{dl}$, triglycerides $\leq 150 \mathrm{mg} / \mathrm{dl}$, systolic and diastolic blood pressure $\leq 90^{\text {th }}$ percentile, and a measure of glycemia to define MHO status.

\section{RISK OF TARGET ORGAN INVOLVEMENT}

Structural and functional cardiovascular modifications including left ventricle hypertrophy, systolic/diastolic dysfunction, and increased carotid intima-media thickness have been considered preclinical indices of CVD in pediatric and adult obese subjects (9, 10). Nonalcoholic fatty liver disease (NAFLD) is increasing globally, and is currently the most common cause of chronic liver disease among youths worldwide (11). Two research manuscripts focused on organ damage such as cardiovascular complications and NAFLD in obese children. Corica et al. demonstrated a negative effect of childhood obesity on subclinical structural and functional cardiovascular modifications. Particularly, severity of overweight, abdominal obesity, and insulin resistance were the main predictors of cardiovascular remodeling, subclinical myocardial dysfunction, and amount of epicardial adipose tissue. Metabolically unhealthy obese (MUO) patients showed a significant unfavorable cardiometabolic profile compared to MHO subjects. Ting et al. demonstrated that liver steatosis was present in $77.2 \%$ of overweight/obese children, and that those with metabolic syndrome were more likely to have advanced liver fibrosis. Also, among children with NAFLD waist circumference predicted risk of liver fibrosis. Overall, these studies suggest that a distinction between MHO and MUO phenotypes might be useful in planning a personalized follow-up approach in obese youths.

\section{DETERMINANTS AND PREDICTORS OF METABOLIC HEALTH STATUS}

The distribution of adipose tissue has a critical role in the metabolic health status. There is an emerging evidence that ectopic adipose tissue deposition is a strong predictor of MUO phenotype (12). In addition to the articles by Corica et al. and Ting et al. pointing to abdominal fat accumulation as predictor of systemic metabolic changes, another point of interest is the article by Payab et al. who showed that in 4,200 children, hip, neck, and wrist circumferences were significantly associated with obesity phenotypes and their metabolic status. These measures may represent innovative, lowcost, and alternative tools for assessing obesity and metabolic syndrome in children. Genovesi et al. in a large pediatric population showed that non-traditional cardiovascular risk factors such as serum uric acid, homeostasis model assessment of insulin resistance index, and waist-height ratio values were independent predictors of being MUO, suggesting that the current definition of MHO may lead to an underestimation of the number of obese children actually at risk for early organ damage.

Hepatic fat content is also an important estimate of systemic metabolic health (12). The identification of potential biomarkers of NAFLD and metabolic syndrome is an important clinical agenda. The study by Mörwald et al. revealed that magnetic resonance imaging-assessed liver fat content and high-sensitivity C-reactive protein best predicted serum ferritin values. These results suggest that ferritin may serve as a marker of early fatty liver disease in childhood obesity, at least in males. This is of interest, as noninvasive surrogate scores such as the fatty liver index have been shown to poorly predict liver fat content in obese children.

There is another aspect which is worth mentioning: the inverse association of vitamin D with obesity, metabolic syndrome, and insulin resistance, in light of its numerous extra-skeletal roles and functions. Esmaili et al. found that vitamin D status and metabolic health display a significant interaction in children and adolescents. The prevalence of hypovitaminosis D was markedly higher among MUO, followed by metabolically unhealthy non-obese, and MHO groups.

\section{ROLE OF LIFESTYLE FACTORS}

Bluher and Schwarz first suggested that lifestyle factors, such as level of physical activity or cardiorespiratory fitness, may play a key role in determination of MHO phenotype (13). Accordingly, a recent systematic review and meta-analysis (14) showed that MHO individuals are more active, spend less time in sedentary behaviors, and have higher level of cardiorespiratory fitness than MUO subjects. In particular, among children and adolescents (14), higher levels of moderate-to-vigorous physical activity, higher cardiorespiratory fitness levels as well as a greater reduction in sedentary behaviors are present in MHO versus MUO children (15-18). Furthermore, in the present Research Topic, Mayerhofer et al. showed that multidisciplinary intervention including exercise, nutritional counseling, and psychotherapy significantly improved body composition, insulin resistance, and inflammation in obese youths.

\section{CONCLUSIONS}

This Research Topic provides an important and timely update on metabolic phenotypes associated with obesity in youths. Future large prospective studies are needed to examine the transition between $\mathrm{MHO}$ and $\mathrm{MUO}$, and the interplay between genetics and lifestyle factors in both development and reversal of such phenotypes.

\section{AUTHOR CONTRIBUTIONS}

CC, LP, BX, and CC-S, conceptualized, designed, wrote and approved the Editorial. All authors contributed to the article and approved the submitted version.

\section{FUNDING}

This work was in part supported by National Natural Science Foundation of China $(81722039,81673195)$ and by the Spanish Ministry of Science and Innovation (CC-S, FJC2018-037925-I). 


\section{REFERENCES}

1. Ajala O, Mold F, Boughton C, Cooke D, Whyte M. Childhood predictors of cardiovascular disease in adulthood. A systematic review and meta-analysis. Obes Rev (2017) 18:1061-70. doi: 10.1111/obr.12561

2. Llewellyn A, Simmonds M, Owen CG, Woolacott N. Childhood obesity as a predictor of morbidity in adulthood: a systematic review and meta-analysis. Obes Rev (2016) 17:56-67. doi: 10.1111/obr.12316

3. Phillips CM. Metabolically healthy obesity: definitions, determinants and clinical implications. Rev Endocr Metab Disord (2013) 14:219-27. doi: 10.1007/s11154-013-9252-x

4. Blüher M. Metabolically Healthy Obesity. Endocr Rev (2020) 41:405-20. doi: 10.1210/endrev/bnaa004

5. Li S, Chen W, Srinivasan SR, Xu J, Berenson GS. Relation of childhood obesity/cardiometabolic phenotypes to adult cardiometabolic profile: the Bogalusa Heart Study. Am J Epidemiol (2012) 176(Suppl 7):S142-9. doi: $10.1093 / \mathrm{aje} / \mathrm{kws} 236$

6. Zhao M, López-Bermejo A, Caserta CA, Medeiros CCM, Kollias A, Bassols J, et al. Metabolically Healthy Obesity and High Carotid Intima-Media Thickness in Children and Adolescents: International Childhood Vascular Structure Evaluation Consortium. Diabetes Care (2019) 42:119-25. doi: $10.2337 / \mathrm{dc} 18-1536$

7. Heshmat R, Hemati Z, Payab M, Hamzeh SS, Motlagh ME, Shafiee G, et al. Prevalence of different metabolic phenotypes of obesity in Iranian children and adolescents: the CASPIAN V study. J Diabetes Metab Disord (2018) 17:211-21. doi: 10.1007/s40200-018-0363-5

8. Damanhoury S, Newton AS, Rashid M, Hartling L, Byrne JLS, Ball GDC. Defining metabolically healthy obesity in children: a scoping review. Obes Rev (2018) 19:1476-91. doi: 10.1111/obr.12721

9. Csige I, Ujvárosy D, Szabó Z, Lőrincz I, Paragh G, Harangi M, et al. The Impact of Obesity on the Cardiovascular System. J Diabetes Res (2018) 2018:3407306. doi: 10.1155/2018/3407306

10. Cote AT, Harris KC, Panagiotopoulos C, Sandor GG, Devlin AM. Childhood obesity and cardiovascular dysfunction. J Am Coll Cardiol (2013) 62:1309-19. doi: $10.1016 /$ j.jacc.2013.07.042

11. Rinella ME. Nonalcoholic fatty liver disease: a systematic review. JAMA (2015) 313:2263-73. doi: 10.1001/jama.2015.5370
12. Neeland IJ, Ross R, Després JP, Matsuzawa Y, Yamashita S, Shai I, et al. Visceral and ectopic fat, atherosclerosis, and cardiometabolic disease: a position statement. Lancet Diabetes Endocrinol (2019) 7:715-25. doi: 10.1016/S2213-8587(19)30084-1

13. Blüher S, Schwarz P. Metabolically healthy obesity from childhood to adulthood - Does weight status alone matter? Metabolism (2014) 63:108492. doi: 10.1016/j.metabol.2014.06.009

14. Ortega FB, Cadenas-Sanchez C, Migueles JH, Labayen I, Ruiz JR, Sui X, et al. Role of Physical Activity and Fitness in the Characterization and Prognosis of the Metabolically Healthy Obesity Phenotype: A Systematic Review and Meta-analysis. Prog Cardiovasc Dis (2018) 6:190-205. doi: 10.1016/j.pcad. 2018.07.008

15. Cadenas-Sanchez C, Ruiz JR, Labayen I, Huybrechts I, Manios Y, GonzálezGross M, et al. Prevalence of Metabolically Healthy but Overweight/Obese Phenotype and Its Association With Sedentary Time, Physical Activity, and Fitness. J Adolesc Health (2017) 61:107-14. doi: 10.1016/j.jadohealth. 2017.01.018

16. Camhi SM, Waring ME, Sisson SB, Hayman LL, Must A. Physical activity and screen time in metabolically healthy obese phenotypes in adolescents and adults. J Obes (2013) 2013:984613. doi: 10.1155/2013/984613

17. de Winter M, Rioux BV, Boudreau JG, Bouchard DR, Sénéchal M. Physical Activity and Sedentary Patterns among Metabolically Healthy Individuals Living with Obesity. J Diabetes Res (2018) 2018:7496768. doi: 10.1155/2018/ 7496768

18. Prince RL, Kuk JL, Ambler KA, Dhaliwal J, Ball GD. Predictors of metabolically healthy obesity in children. Diabetes Care (2014) 37:1462-8. doi: $10.2337 / \mathrm{dc} 13-1697$

Conflict of Interest: The authors declare that the research was conducted in the absence of any commercial or financial relationships that could be construed as a potential conflict of interest.

Copyright (c) 2020 Chiesa, Pacifico, Xi and Cadenas-Sanchez. This is an open-access article distributed under the terms of the Creative Commons Attribution License (CC BY). The use, distribution or reproduction in other forums is permitted, provided the original author(s) and the copyright owner(s) are credited and that the original publication in this journal is cited, in accordance with accepted academic practice. No use, distribution or reproduction is permitted which does not comply with these terms. 